\title{
Los Cantos de Kanaima
}

\author{
Matthias Lewy \\ Instituto de Artes (IdA) da Universidade de Brasília, Brasília, Distrito Federal, Brasil \\ E-mail: matthiaslewy@gmail.com
}




\section{Resumen}

En primer lugar, la atención se centra en la clasificación de la entidad Kanaima en el multiverso del grupo indígena Pemón (Venezuela/Brasil). En segundo lugar, se presentan las grabaciones históricas y actuales de las canciones de Kanaima, en las que se examinan las funciones de estas canciones con respecto a la administración del cuerpo/alma y los procesos de (re) transformación asociados. Finalmente, se discute la peculiaridad de esta entidad anti-humana en las Guayanas en comparación con las ontologías animistas de la Amazonía.

Palavras-chave: Las Guayanas. Pemón. Música Indígena. Kanaima.

\section{Abstract}

Firstly, the focus is on the classification of the entity Kanaima in the multiverse of the indigenous group of Pemón (Venezuela / Brazil). Secondly, the historical and current recordings of Kanaima songs are presented, whereby the functions of these songs are examined with regard to the body/soul administration and the associated (re)-transformation processes. Finally, the peculiarity of this anti-human entity in the Guianas is discussed in comparison to the animistic ontologies of the Amazon.

Keywords: Guianas. Pemón. Amerindian Music. Kanaima. 


\section{Introducción}

M.L.: El Kanaima es un Pemón?

A: Si claro, bueno.. o sea.. pero cuando quiere.. esa transformación.. y cuando mata... se queda sin piedad $y$ sin alma pues.

Florinda no le gusta lo que está escuchando: "¿Quién canta eso?",
me pregunta. "No sé", le respondo. Me siento incómodo, con un montón de preguntas en mi cabeza. “Tendría que ver la información de Koch-Grünberg", le digo. Rápidamente busco el archivo que me mandaron del Phonogramm-Archiv de Berlin. "Aquí dice: cilindro número 13 con el título oareba, cantan dos hombres, y que se baila en la noche". "Es mala, la canción", me dice ella.

Balbina interrumpe, diciendo que oareba "es el warepan de nosotros Pemón, un baile que es divertido normalmente".

"No sé", murmura Florinda y sigue diciendo: "Esta canción no es divertida, para nada. No se escucha que cantan aiyan, aiyan, aiyan otra vez? Como en la canción de antes también."

"Si", dice Balbina, "esa también dice aiyan" y Florinda confirma: "Si, el aiyan de murúa también es malo. Todos son cantos de un tiempo cuando cantaban a los makoi, a los malos, son cantos de Kanaima."

Esta comunicación fue parte de una reunión sonora con especialistas de la cultura Pemón ${ }^{1}$ en la que escuchamos los cilindros de cera de Theodor Koch Grünberg en una comunidad Makuxí/Taurepán ubicada en la frontera entre Brazil y Venezuela, en el año 2015. El lingüista y antropólogo alemán grabó 86 cilindros en el año 1911 en esta región. Hoy en día las prácticas de la música indígena Pemón como Theodor 
Koch-Grünberg las observó y grabó siguen existiendo en la memoria de los indígenas más viejos. Por ello, este texto presenta consideraciones surgidas en reuniones sonoras con diferentes especialistas Pemón, así como con otras personas interesadas en las grabaciones.

Esa reunión con Florinda y Balbina fue la primera ocasión en que escuché hablar de los cantos específicos de los kanaimaton.

\section{2 ¿Qué es un kanaima?}

El tema de los kanaimaton (plural) es heterogéneo y ambivalente. El único consenso que se encuentra en el discurso académico describe a esta entidad como un asesino. Varían las descripciones de los motivos y las formas de matar, como también del papel del kanaima en la sociedad indígena.

Históricamente el termino kanaima refiere a otros grupos étnicos que eran considerados salvajes u hostiles. En las entrevistas siempre aparece el conflicto histórico entre los Pemón-Arekuna y Makuxí, conflicto que también es mencionado en las obras de Koch-Grünberg (1923, p. 102, según Robert Schomburgk, 1841, p. 366-379 y Richard Schomburgk, 1848, p. 147.) Este conflicto tradicional se transmitió hasta hoy en día. Las comunidades de Kerepakupay merú (San Francisco de Yuruaní) que son dominados por Pemón-Taurepán acusan a los vecinos de la comunidad Makuxí de San Ignacio por ser kanaimaton y vice verse. Pero también los habitantes de la comunidad Paraitepuy cerca de Kerepakupay meru tienen la fama de kanaimaton. Los cazadores de kanaima también se dedican a la caza de personas sobre las que existe la sospecha de que tienen prácticas de kanaima. Esto muestra la complejidad de los usos del concepto kanaima que tiene también un rol en la economía cuando utilizado para desacreditar a posibles competidores.

Algunas veces el término es usado para denominar a personas de mal carácter, que roban o engañan a la gente. Es, a veces, una forma de humillación que puede generar acusaciones falsas, denunciando como asesinos a personas inocentes. Esos ejemplos explican porqué 
para un observador externo es difícil comprender el fenómeno sin investigar profundamente todos los procesos e interacciones sociales.

El análisis de los cantos de los kanaimaton de esta etnografía se aproxima a preguntas ontológicas como:

¿Qué tipo de entidad es un kanaima?

¿Por qué razón esta entidad mata y a quién?

La pregunta por la cualidad de la entidad de un kanaima es compleja. La mayoría de los discursos antropológicos subrayan que es un ser humano, que tiene la capacidad de transformarse en animal y/o de actuar solamente con su espíritu.

David Thomas (1982, p. 160) presenta seis definiciones que describen el uso de la palabra kanaima en el área de los Pemón:

1. los kanaimaton son seres que se manifiestan en forma humana o animal; ejercen control sobre los seres humanos vivos e imponen su voluntad sobre ellos;

2. kanaima es la causa actual o final de todas los enfermedades serias y de la muerte;

3. kanaima puede referir a una entidad espiritual pura o a la encarnación de un espíritu en forma humana o animal; individuos de procedencias no-Pemón están imputada de ser kanaimaton; los kanaimaton son mandados por chamanes o los chamanes mismos son kanaimaton;

4. los kanaimaton se encuentran más en la selva que en la sabana y les gusta actuar en la noche.

Ese resumen de Thomas es una primera introducción al tema que nos deja con algunas preguntas. Basándome en mi experiencia se constata que los kanaimaton no se limitan a otras comunidades o a los no-Pemón, tampoco es un fenómeno limitado a la selva y a la noche. Otro aspecto problemático refiere al proceso de transformación del cuerpo y/o del espíritu y las formas de interacción con los piasan (chamanes, Pemón). 


\section{Transformación}

Civrieux nota en el caso de los Yekuana que los kanaimaton no son espíritus sino seres humanos con la capacidad de transformarse en monstruos, tigres o serpientes. Los Yekuana negociaron con los grupos Caribes y Arawakos hasta la región de alto Essequibo donde se afectaron con kanaima y fueron recuperados for los vecinos Kariña, que ayudaron con sus chamanes (Civrieux, 1980, p. 172; Halbmayer, 2010, p. 87).

Audrey Butt Colson traduce el término kanaima como el "gran terminador" (Butt Colson, 2001, p. 224) o "asesino secreto" usado entre los Pemon, Kariña y Lokono (Arawak), mientras que los Akawaio usan itoto (Butt Colson, 2001, p. 221). Por un lado la antropóloga inglesa dice que el kanaima mata en su propia persona, es decir, como ser humano (Butt Colson, 2001, p. 223). Por otro lado, ella precisa el proceso de la transformación:

Además, se le considera capaz de separar su fuerza vital, o espíritu, de su cuerpo y de actuar como asesino desencarnado. Akawaio y Pemong dicen que de esta manera él puede ser visto como una persona ordinaria en un lugar que es mandado por su maldad inherente y "mente malo". Existen sustancias de plantas especiales (notable de la especies kumi, que son carrizos y yerbas gruesas) que facilitan Itoto (Kanaima) a viajar muchas millas en pocos minutos. Todo esto es coherente con la creencia general en que todas las formas de vida consisten de un cuerpo y una fuerza vital o espíritu residente (Akawaio, akwalu, Pemong, ekatong). La fuerza vital sale de su cuerpo por un tiempo durante los estados de inconsciencia: sueños, desmayos o en coma. Un chaman separa su espíritu deliberadamente cuando esta alucinando por tabaco. Itoto (Kanaima) puede separar voluntariamente su fuerza vital y mandarlo para matar, pero también él tiene la opción de mandarlo [su fuerza vital] a otra criatura, un perro, pájaro, jaguar, venado, etc., usando su cuerpo como un disfraz. ${ }^{2}$ (Butt Colson, 2001, p. 223)

Además la autora nota que el kanaima representa una antiestructura de la vida social (Butt Colson, 2001) diciendo que los kanaimaton son solamente hombres que viven fuera de la comunidad 
humana para atacar seres humanos con la excepción de sus propios parientes ${ }^{3}$.

Estas informaciones no fueron confirmadas por parte de los especialistas Pemón a quien consulté. Para ellos los kanaimaton pueden atacar a miembros de su propia familia, no necesariamente tienen su casa fuera de una comunidad, y las mujeres también pueden actuar como kanaimaton.

La explicación del viaje del alma (yekaton/ekatong según Butt Colson) se confirma en el trabajo de Whitehead (2002). El autor presenta un análisis histórico completo con datos de campo propios.

Según Whitehead el kanaima también es una persona, que puede aparecer como persona o como jaguar en la selva, o como un hormiguero en la sabana (Whitehead, 2002, p. 89).

Debe destacarse que Whitehead (2002, p. 108) incluye una entrevista con un kanaima en su presentación de los "chamanes oscuros". El protagonista habla sobre su intención de matar seres humanos:

Kanaima es más antigua que piya [chamán, nota del autor), más antigua que la guerra. Buscamos nuestra alimentación. Eso es todo, eso es todo. Buscamos a alguien y cuando vemos algo dulce para chupar y todo eso [procedimiento ritual], es para la alimentación. Se impulsa su espíritu por arriba, arriba para ir lejos a kalawali ${ }^{4}$. (Whitehead, 2002, p. 109)

Esa frase es fundamental para el análisis del fenómeno de kanaima como vamos a ver más adelante.

Para acerarnos a una respuesta sobre las formas de transformación del kanaima, su cualidad de entidad y su intención de matar se puede incluir una pregunta importante: ¿Qué tiene que ver el kanaima con la grabación de oareba de Koch-Grünberg?

\section{Las Fases de Kanaimaton y el rol del Sonido}

Si queremos saber qué papel juega el sonido o la música en este fenómeno tenemos que analizar la forma de existencia de los kanaimaton. 
Observamos que ningún autor ha mencionado hasta ahora la existencia de una música kanaima.

Whitehead nota una gran separación entre "piya" (chaman/ Patamona) "kanaima" y "alleluya" (Whitehead, 2002, p. 99). Según este autor los tres grupos representan tres categorías diferentes definidas por categorías como "modo sensual, performance sensual, recursos mágicos, equipos mágicos, sustancias mágicas, performance del ritual, lugares del ritual, domicilio de los espíritus" y el "patrón de los espíritus".

Mientras los representantes de alleluja tienen como modo sensual la canción y el olfato, el chaman esta representado por su visión y el kanaima por sus capacidades extraordinarias de olfatear, de oír y de ver.

Lo más interesante es la categoría "performance del ritual" (ritual performance). Whitehead anota para el chaman un "control del espíritu" ( spirit control) mientras que el kanaima se define por su "cambio de forma" (shape shifting, Whitehead, 2002, p. 99).

Esa comparación remite a la problemática del kanaima evidenciado en el peligro de "perder" su alma (yekatón) durante el proceso, después del asesinato.

A partir de la comparación de mis datos de campo con la literatura mencionada se constata que el asesinato del kanaima se organiza en tres etapas. La primera etapa se caracteriza por la persecución de la víctima y el maltrato. La segunda es el acto de matar, y la tercera se define por la re-transformación.

Para entender mejor esas tres etapas conviene considerar que el kanaima se manifiesta como Pemón en la vida cotidiana y en las fases en que él no esta matando, es decir, el kanaima es un ser humano con alma y cuerpo. Pero en la fase de planificación y realización de un asesinato cambian las cualidades de la relación alma/cuerpo. Por esa razón propongo diferenciar las distintos cualidades y acciones. Kanaima como término no es un sustantivo, sino un verbo (una acción) que puede usarse como "hacer kanaima", o para definirlo como una fase temporal, propongo "estado de kanaima".

De esta manera podemos deducir tres fases o cualidades de "estado de kanaima".

De esta manera podemos deducir tres fases o cualidades de "estado de kanaima". 
Las tres fases y cualidades de "estado de kanaima" son :

1. perseguir, maltratar - persona/vuelo de alma;

2. matar, en el momento concreto de asesinato se da la transformación en animal/monstruo sin alma;

3. re-transformación, después de matar, estado de locura y calentamiento/necesidad de recuperar el alma;

La primera etapa fue descripta de forma detallada por Butt Colson (2001), Thomas (1982) y Whitehead (2002). Mis datos de campo confirman esos resultados. En el hospital de Santa Elena es bastante conocido el ataque de kanaima a pacientes indígenas.

El kanaima prefiere indígenas Pemón que caminan solos en la sábana. También se acerca a las casas. Tiene un silbato típico que produce miedo a las víctimas. Los daños físicos son causados por el uso de venenos de diferentes plantas y serpientes. Durante los primeros ataques el kanaima usa sustancias que producen una inflamación de la lengua con el resultado de que la víctima no es capaz de hablar ni comunicarse con sus familiares. Estos ataques iniciales producen fracturas óseas en las manos seguidas por inserciones de diferentes yerbas y hojas por el ano y oralmente. La meta es producir inflamaciones seguidas de fiebre para secar el cuerpo por adentro. Las víctimas sufren durante tres días o hasta una semana y mueren al final. No obstante, la fase siguiente puede durar algunos años.

En la primera fase del ataque el kanaima aparece como un ser humano, aunque también puede usar la técnica de transformarse en un animal. En este contexto el kanaima aplica una fórmula mágica (tarén), y su kumi (planta mágica), para transformarse. El proceso de la transformación no significa necesariamente que un alma (yekatón) de kanaima entra en un animal como un jaguar (o en un hormiguero). Es el tarén que, junto con el kumí, cambia la perspectiva en el mundo de los seres humanos, no solo de la víctima sino de todos los Pemón. Por ello, pueden encontrarse las huellas de un jaguar o de un hormiguero, es decir, estos seres dejan sus evidencias en el mundo de serewarö, que es el mundo presente compartido por los seres humanos. No esta bien definido qué es el alma de un kanaima que mata. 
Cuando la víctima muere el kanaima también sufre, Es la "locura", descripta como la enfermedad de los kanaima.

Puede constatarse que la víctima y el asesino están conectados por su físicalidad, no por sus interioridades. También es importante darse cuenta que todos los kanaimaton son conectados entre sí. Cuando uno de ellos mata, todos sufren un malestar físico.

Este reconocimiento se basa en un análisis del tratamiento que un chamán realizó con una niña de doce años. Sus padres estaban preocupados por la salud mental de la niña, porque de repente ella empezó de hablar en voz baja ('como un hombre'), a fumar tabaco, a subir a las paredes de la casa y a decir locuras. El primer diagnóstico del chaman fue que, a lo mejor, la niña es una profeta de los orekotón (el colectivo areruya). El chamán mandó a la niña a un grupo de orekotón que practicaban los rituales areruya y cho'chiman, pero ellos negaron la hipótesis del chamán porque la niña empezó de comportarse mal y a subir a las paredes de la cho'chi durante el ritual. Después el chamán se dio cuenta que la interioridad (el alma) de la niña había sido secuestrada por el espíritu de una serpiente, ante el que nada pudo hacer. Su nuevo diagnóstico decía que la niña era una kanaima. Ella misma, finalmente, confirmó la declaración del chamán diciendo que siempre tenía esos ataques físicos cuando un kanaima mata. Además, había empezado a sembrar kumí y tabaco sin tener experiencias o enseñanzas previas. Así confirmó a su familia y al chamán que ella tendría este "defecto mental", como son conocidas entre los Pemón las conexiones físicas con los kanaima. Finalmente la familia decidió sacarla del territorio y alejarla de influencias indígenas, llevándola a una ciudad criolla. Hoy en día esta recuperada y actúa en sincronía con su espíritu y su cuerpo.

La pregunta por la interioridad y/o el alma es clave para acercarse al fenómeno del estado kanaima.

En el momento de la muerte, en que el alma de la víctima no puede regresar al cuerpo, el kanaima siente esa conexión, no con su alma sino con su cuerpo. El cuerpo del kanaima se transforma en un "monstruo" y su conciencia no funciona bien por falta de las cualidades del alma. Se calienta y se "vuelve loco", según los indígenas.

Este momento de estado de kanaima es el más peligroso debido a la falta del alma. El kanaima tiene que recuperar su alma. Para ello 
existen distintos métodos. El primero es un acto de antropofagia como se describe en un mito grabado por el misionero Cesáreo de Armellada. Fue publicado con el título: "Un indígena que vio al Awoinorü'pö."5 (Armellada. 2013, p. 229, I):

Un indígena se fue de viaje en casa de sus compañeros. Pero, llegado allá, se encontró con que estaban muertos y enterrados en la misma casa y los otros se había ido.

No obstante, el indígena posó allí con sus dos hijas, colgando muy altos sus chinchorros. Aquella noche, estando el indígena acostado arriba, llegó el awoineripué. Entró en la casa donde estaban enterrados los indígenas. Con una varita puyó los sepulturas y probó diciendo: “¿Ya se enfuertó la bebida del kanaimü?" [¿Ya se fermentó la bebida del kanaimü?, nota del autor].

De la misma manera fue probando de todas las sepulturas diciendo:" ¿Ya se fue enfuertó la bebida del kanaimü?"["¿Ya se fermentó la bebida del kanaimü?", nota del autor].

Después que hubo probado de todas sepulturas, se puso a buscar su pito diciendo: “¿Está aquí el piro del kanaimü?"["¿Ya se fermentó la bebida del kanaimü?", nota del autor].

Cuando lo encontró, alegre porque su bebida empezaba a enfuertarse (los cadáveres estaban en descomposición), se puso a bailar alrededor de las camazas de su kashirí (las sepulturas) y tocaba su pito:"isein, tirirí; sein, tirirín!".

Después de tocar su pito, salió fuera ver si venían sus compañeros al baile, dejando allá su pito. Entonces el indígena se precipitó de un salto al suelo y asió el pito; y volvió a encaramarse en su chinchorro.

Diciendo:" ¿Está aquí el piro del kanaimü?", el awoineripué volvió a entrar en la casa, se puso a buscarlo, pero no lo halló.

En este llegaron sus compañeros: venados, dantos, tigres, zorros y toda clase de animales nocturnos, que él había convidado. Y el awoineripué le dijo: "Búsquenme el pito, que se me perdió".

Entonces todos aquellos bichos y animales se pusieron a bailar y cantaban a coro diciendo: "Este, éste; lo encontré". Pero nada que aparecía.

Pero entonces el indígena tocó una camaza vieja, que había 
colgada del techo. Con este ruido los bichos cayeron en tierra, y después echaron a correr despavoridos.

Cuando lo bichos huyeron, el indígena arrebujó a toda prisa su chinchorro y el de sus hijas y se regresaron a su casa corriendo.

Pero el awoineripué les fue siguiendo los pasos por causa de su pito.

Al día siguiente, antes de irse al conuco, el indígena escondió el pito en la cumbrera de la casa entre la palma, y les dijo a sus hijas: "Si viene el awoineripué, no les muestran su pito".

Después que el indígena se había ido al conuco el awoineripué se llegó calladamente y se arrimó a la pared de la casa.

En este momento la niña más chiquita estaba llorando y para acallarla, su hermana mayor le dijo: "Cuidado no venga el kanaimü a buscar pito, que escondió papá en la cumbrera de la casa".

Entonces el awoineripué entró y les preguntó: “¿Qué es lo que estaban diciendo?". Nada -respondió la hija del indígena-, pues, pues, pues yo decía que mi papá me había armado esta casa".

“iQué va! -dijo el awoineripué-tú estás diciendo al revés. ¿Acaso no te oí decir: el pito que escondió papá en la cumbrera de la casa? Ya me lo están mostrando o si no, les meto veneno en la boca".

Entonces la indígena, mostrándoselo con la mano, le dijo: "Míralo allá". Y el awoineripué se encaramó hasta la cumbrera de la casa y se lo llevó.

Cuando recobró su pito, el awoineripué les arrojó veneno (werupö) a las hijas del indígena en la boca y se alejó corriendo, y tocando su pito: iSein, tirirí; sein, tirirín!". El indígena regresó del conuco, pero encontré a sus hijas muertas.

Las explicaciones del misionero católico son contradictorias. Por un lado escribe que la base de la narración es la creencia en la existencia de los kanaimaton. Se refiere a la entidad con el nombre Awoineripué caracterizándola como una "figura" con "ojos grandes y brillantes" (Armellada, 2013, p. 232, I). Por otro lado se encuentra en el diccionario de Armellada que Awoineripué es un: "ser fantástico, 
que se alimenta de los cadáveres, y de hábitos nocturnos. A veces se le confunde con los Kanaima." (Armellada, 2007, p. 26)

Este confusión del misionero refleja los diferentes pensamientos sobre este ser. Mis informantes tampoco confirmaron si Awoineripué es una forma de kanaima. Tampoco sabían si los kanaimaton juegan con sus víctimas después del entierro con sus pitos, como esta descripto en el mito de Armellada. A su vez, Whitehead menciona exactamente esa acción del kanaima:

Cuando la tumba es descubierta [por el kanaima, nota del autor], se insertaun palo, a través del suelo, hasta el cadáver. Después el palo es retirado y se chupa la maba, un jugo con sabor a miel. Dicen que los jugos de la putrefacción tienen sabor a miel, por ello se "prueba" la tumba con la ayuda de un palo, de la misma manera que al buscar miel en una colmena. El efecto de la maba es psicotrópico y mórfico: el Kanaima satisfecho puede, como un jaguar, dormir saciado y soñar como una persona. El sueño también es una realidad en que el jaguar se transforma en persona a causa de haber probado la maba. Así un Kanaima no va a alimentarse con comida de seres humanos antes de que haya probado la comida sagrada de la tumba. Además tiene que probar la maba para eliminar las peligrosas fuerzas divinas del Señor Jaguar y para regresar al ámbito humano ${ }^{6}$. (Whitehead, 2001, p. 238)

La idea de un Señor Jaguar no se encuentra en el mundo de los Pemón hoy en día, tampoco esa forma de antropofagía. Por un lado se puede leer el mito de Awoineripué como una referencia a una memoria cultural que se olvidó, cambió o que siempre fue diferente en la práctica de los kanaima, dependiendo de las diferentes perspectivas Pemón comparadas a las de los Patamona. Por otro lado hay similitudes y paralelismos en el hábito, en la intención y, lo más importante, en la forma de la re-transformación de los kanaimaton.

Debe subrayarse que en la explicación de Whitehead aparece la idea de que el kanaima necesita la maba para su re-transformación en un ser humano. Es la razón por la cual Awoineripué se concentra en la recuperación de su pito en el mito de Armellada.

El asunto de esta re-transformación se encuentra también en el concepto de kanaima en el mundo Pemón. Según mis informaciones 
el kanaima se transforma en un "bicho" o "monstruo" caliente y loco en el momento directo del asesinato, es decir, en la situación en que el cuerpo de la víctima no es capaz de recuperar su alma a causa del maltrato por parte del kanaima.

En esta fase de ser un monstruo caliente y loco el kanaima es muy vulnerable y los parientes de la víctima tienen una buena posibilidad para una venganza. El kanaima por su parte tiene diferentes opciones para calmarse, enfriarse y finalmente re-transformarse en un Pemón. En este momento también tiene una conexión con una realidad diferente (serewarö) en la cual también esta actuando, pero este mundo no es descrito como un mundo de sueño o de un "Señor Jaguar", como en el caso de los Patamona de Whitehead.

En la cosmología Pemón este mundo se llama "aiyan" y para entrar e interactuar con las entidades en este mundo se debe cantar.

\section{El Multiverso Pemón}

Las palabras aiyan sirven para que el kanaima entre a este estrato del multiverso Pemón. El concepto de mundo en la cosmología Pemón contiene diferente estratos que forman un multiverso (Halbmayer, 2010). Aparte del serewarö (aquí y ahorra/realidad), el pia daktai (comienzo y fin/mundo mítico) y el wakü pata (paraíso/mundo de los orekotón) se debe adicionar el estrato de aiyan.

Estratos son colocado en distintos partes del paisaje como una área invisible pero audible, habitada por entidades específicas. Es sentido común entre los pemón que los seres humanos comparten el mundo con todo tipo de animales. El lugar de los animales de presa como tapires o jabalíes es tan importante como el de los peces. Todos estos animales viven y tienen su lugar en el serewarö.

También los espíritus (mawaritón) viven en las montañas (tepuy), que son sus casas. Otras entidades son los espíritus del agua de la familia rató. Según la cosmología Pemón existen entidades que no son visibles por los Pemón normales, pero sí son audibles. Son los maikok que ocupan diferentes áreas en la sabana y marcan su territorios con telarañas o con un sonido de un pájaro (wantoto) para avisar a los seres 
humanos que no deben pasar por su territorio sin dejar una ofrenda para ellos. La apropiación del concepto paraíso refleja la idea que hay un lugar en que viven las almas de fallecidos de las familias Pemón que practicaron los rituales de los orekotón ${ }^{7}$ (areruya, cho'chiman). Cada fin de semana se encuentran los orekotón vivos durante la performance de dichos rituales.

Las entidades de estos estratos deben conectarse a través de canciones que realizan una interacción en el pia daktai. Por ejemplo el pia'san canta a los mawaritón usando canciones que recibió en este mundo, los cazadores de jabalíes o tapires cantan al dueño (esak/chiwön) para atraer su presa. De la misma manera los pescadores cantan al rató como dueño de los peces (Lewy, 2012).

Estas descripciones refieren apenas a algunos aspectos de un multiverso complejo. Puede mencionarse que una característica del multiverso Pemón es su flexibilidad para el cambio ${ }^{8}$. No obstante, falta todavía una parte importante de esta presentación que es la del estrato de aiyan.

\section{El Estrato de Aiyan}

En la literatura aparece este término en el contexto de los bailes de aiyan. Butt Colson y Armellada (1989, p. 172; Halbmayer, 2010, p. 192 ) escriben que aiyan es el nombre de un baile entre los Pemón que es el imawari entre los Kapón. El termino aiyan significa oscuridad y debilidad y presenta un concepto opuesto al la idea auka (Lewy, 2011) que es la luz y la fuerza de vida.

El baile era ejecutado durante la temporada de la seca con la intención de pedir frutas, animales de presa y peces:

Parece que la actividad particular de los Kapon y Pemón se refería a la base de la cadena alimentaria, al desarrollo vegetativo y al ciclo de floración de la siembra y cosecha de las frutas que soporten el imperio de los animales en general - sobre la que todo depende, ya sea directo (a través de comer frutas) o indirecto (a través de de comer aquellos que comen frutas) ${ }^{10}$. (Butt Colson; Armellada, 1989, p. 172; Halbmayer, 2010, p. 192) 
Se debe constatar que estas entidades del "imperio de los animales" representan otra metáfora del mundo del "señor Jaguar" según Whitehead. Según mis informaciones son las entidades del estrato de aiyan.

Además Butt Colson y Armellada mencionan que el ritual de aiyan fue substituido por los rituales de areruya y/o chimitin ${ }^{11}$ que frecuentemente son practicados en la época de navidad (Butt Colson; Armellada, 1989, p. 185; Halbmayer, 2010, p. 192). De acuerdo con este idea parece entendible que los espíritus cristianos fueron apropiados como una nueva fuente segura para conseguir recursos en comparación con las entidades de aiyan. Por otro lado, puede decirse que estas entidades de aiyan no desaparecieron, por lo menos en los rituales de areruya y cho'chiman.

Discutiendo este asunto con Florinda, una mujer Pemón que practica los rituales y vive según las normas de los orekotón, las fuerzas negativas de la naturaleza están en el mundo makoi. Pude encontrar este concepto también en mis investigaciones sobre el areruya y cho'chiman durante los años 2005-2009 en el territorio de lo Arekuna y Kamarakoto (Lewy, 2011).

Makoi es un estrato cerca de la cho'chi (la casa del ritual) donde está la energía mala del mundo caracterizado como el mundo de los diablos.

Así, puede comprenderse mejor la reacción de Florinda descripta en el inicio de este texto. Según ella, la canción de oareba del cilindro de Koch Grünberg refiere a este mundo. Un mundo con el que se conectaron por un lado los pia'san, y por otro lado los kanaimaton que son finalmente mandados desde este estrato al mundo humano.

Para acercarse a este último asunto de la conexión de los kanaimaton con el mundo de makoi y/o aiyan, se debe analizar la performance sonora de los kanaimaton.

\section{Transcender el Estrato de Aiyan}

La palabra aiyan en su forma cantada refiere a una interacción de un chamán o kanaima con el estrato aiyan. Este estrato no esta habitado según mis informantes con imawari o mawariton como escriben 
Armellada y Butt Colson, sino por entidades de una energía mala pero también necesaria, como vamos a ver más adelante.

En el canto de un chamán en su sesión de cura la palabra es usada en dos contextos. Primero, para llamar a los imawari en el pia daktai y, segundo, para conectarse con las energías del estrato aiyan. En la colección de Cesareo de Armellada en Santa Elena de Uairén se encuentran tres grabaciones de una curación de un chamán con el título murúa. El análisis de la primer parte muestra una interacción con un mawarí. La segunda parte también refiere al mundo de los espíritus sin usar la palabra aiyan. No obstante en la tercera parte el chamán canta y nombra a las entidades ${ }^{12}$ del estrato aiyan repitiendo muchas veces la misma palabra.

Además el murúa era un género cantado durante las festividades en rondas colectivas. El cilindro número 12 de la colección de cantos del murúa de Koch-Grünberg contiene la palabra ayian de la siguiente manera:

\begin{tabular}{|ll|}
\hline 1. tüwotori'pö pona & 1. Después de la cacería \\
2. tarikuyamai & 2. me adorné \\
3. Muroko wadarirü'pe & 3. Así como la voz del pez \\
4. Umanupamüy & 4. luego a la danza, terminé \\
5. Aiyan, aiyan, aiyan & 5. Aiyan, aiyan, aiyan \\
\hline
\end{tabular}

Imagen 1: Cilindro de cera Número 12, Colección de Theodor Koch-Grünberg, Phonogramm-Archiv Berlin VII_W_2819_K_GR_BRASILIEN_12 Fonte: Transcripción/Interpretación: Balbina Lambos y Florinda Infante Hernández (2015)

El texto de este canto (Imagenl) refleja la acción de la preparación para la danza y el trabajo del chamán en un contexto colectivo. Una interpretación será que la "voz del pez" refiere al espíritu del agua, que es rató, porque la forma de cantar, los ornamentos, el ritmo de baile y el texto muestran las características de esta entidad. La palabra aiyan representa un ornamento sonoro en el canto de los chamanes. Su función es la de una "palabra mágica" para abrir los mundos del pia daktai y de aiyan. Se debe constatar que el texto refleja dos acciones, "abrir" uno de los estratos en general y nombrar la entidad específica en este mundo. 
Entonces podemos preguntarnos de qué se trata el oarebá que grabó Koch-Grünberg sin dejarnos explicaciones adicionales.

1. Aiya ada aiya aiya
2. Ada aiya ada aiya
3. Aiya da aiya

Imagen 2: Cilindro de cera Número 13, oarebá según Theodor Koch-Grünberg, warepan según Balbina Lambos y Florinda Infante Hernández, Colección de Theodor

Koch-Grünberg, Phonogramm-Archiv Berlin VII_W_2820_K_GR_BRASILIEN_13 Fonte: Transcripción/Interpretación: Balbina Lambos y Florinda Infante Hernández (2015)

Balbina y Florinda estaban seguras de que el término warepan usado por los Pemón hoy en día corresponde al género de oareba que antiguamente eran cantos de aiyan. Estos cantos son practicados por los pia'san, los kanaimaton y también durante los festividades de aiyan en forma colectiva. Sin embargo el texto del número 13 no nombra ninguna entidad. Tampoco encontramos más informaciones específicas en los pocos fragmentos de las grabaciones de oarebá que son inteligibles por parte de las especialistas en los cilindros de número 5 (Imagen 3), 6 (Imagen 4) y 13 (Imagen 2) y 14 (Imagen 5).

$\begin{array}{ll}\text { 1. Awari makö'dan } & \text { 1. Estas cantando a tu instrumento } \\ \text { 2. Aiyan aiyan } & \text { 2. Aiyan aiyan }\end{array}$

Imagen 3: Cilindro de cera número 5, Colección de Koch-Grünberg, PhonogrammArchiv Berlin VII_W_2812_K_GR_BRASILIEN_5

Fonte: Transcripción/Interpretación: Balbina Lambos y Florinda Infante Hernández (2015)

\section{Inna türüda! 1. Estamos aquí!}

Imagen 4: Cilindro de cera número 6, Colección de Koch-Grünberg, PhonogrammArchiv Berlin VII_W_2813_K_GR_BRASILIEN_6

Fonte: Transcripción/Interpretación: Balbina Lambos y Florinda Infante Hernández (2015)

En el contexto de la grabación de cilindro número 5 (Imagen 3) el término awari (a-tú, pos. y -wari, instrumento) refiere a la voz 
del cantante. Cantar a la voz parece a primera vista una acción auto referencial. Sin embargo en este contexto es una acción de "humanizar" la voz, es decir, que la voz humana genera una entidad propia para conectarse con las entidades del estrato aiyan.

El cilindro número 6 (Imagen 4) muestra que es un canto para baile que también era practicado en grupo como implica el uso de la tercera persona plural, indicado por el pronombre posesivo inna (nosotros).

Este breve resumen de la palabra aiyan muestra que su uso y función dependen del contexto y de la intención. La mayoría de los Pemón usaron la palabra en sus cantos excepto los orekotón, los que practican areruya y cho'chiman. Mientras la performance en grupo tenia la intención de conectarse con el estrato de aiyan para pedir a las "energías de la naturaleza" que crezcan las frutas comestibles, el pia'san y el kanaimaton tenían otra intención.

Con los cantos de aiyan ambos son capaces de entrar al estrato de aiyan. ¿Pero cuál es la intención en comparación con los grupos colectivos específicamente en el contexto kanaima?

Antes de profundizar esa discusión se debe reflexionar sobre el género principal de los kanaimaton que es el amanawui.

\section{Amanwui - el Canto de los Kanaimaton}

Según mis profesores indígenas el género de amanawui tiene dos significados. Por un lado son cantos de amor y por el otro lado son los cantos de los kanaimaton.

Por el momento no vamos a entrar en una discusión sobre esta ambivalencia, sino que analizaremos las dos sub-categorías del amanawui de los kanaimaton.

La primera sub-categoría contiene los cantos que sirven como un sustituto al acto de la antropofagia.

Como hemos visto antes, según las descripciones de Whitehead el kanaima tiene que chupar la maba de su víctima para tener la opción de re-transformación. Según mis informantes este acto es diferente en el mundo Pemón. Chupar jugo de la víctima con un pito no se reconoce como una práctica del kanaima. Sin embargo existe otro acto de antropofagia en la memoria de la cultura Pemón. 
Para la re-transforamción del estado de kanaima, es decir de estar con un cuerpo de un monstruo sin alma, caliente y loco el kanaima tiene que consumir una parte de la carne del muslo de la víctima o, alternativamente, el kanaima puede cantar un amanawui para enfriarse.

Nótese que a través del canto el alma de la 'persona' kanaima entra nuevamente en el cuerpo. De este manera se re-transforma el "bicho" o "monstruo" en un Pemón, es decir una persona con cuerpo humano y alma humana. Entonces surge la pregunta sobre cómo funciona esta re-transformación a través de un canto.

Existe una clase de amanawui que usan las kanaima para calentarse, el canto sirve para que baje la temperatura del cuerpo y así la posibilidad que entre el alma al cuerpo otra vez. La Imagen 5 contiene un texto de un amanawui que he grabado en el territorio Arekuna. El texto refleja la acción de la primera fase de la transformación en una sirena del mar, que es un estado preliminar para re-transformarse finalmente en un Pemón. El cantante se ubica en este momento en el estrato de aiyan, en que todos los kanaimaton están conectados de una manera. Así todos se sienten tocados físicamente cuando uno de ellos (kanaima) mata y esta en la fase de re-transformarse, necesitando refrescarse.

Parau saru uyau En el corriente del oceano,

tuma sewasurumai tunakoto ${ }^{13}$ al lugar de las aguas, me transformo en una sirena.

Imagen 5: canción de amanawui

Fonte: Grabación de Matthias Lewy, archivo privado, Transcripción/Interpretación: Balbina Lambos

\section{La Intención de Kanaima}

En muchas publicaciones leemos que un kanaima es una persona mala. Sin embargo mis informantes me ofrecieron una explicación diferente. El estrato de aiyan es la fuente de la esencia de la vida. Es la fuente de todas las cosas que crecen y así el gran proveedor de la comida para el ser humano. Para este "don" o regalo las entidades del estrato de aiyan exigen algo en el proceso de la reciprocidad. Los entidades que aparecen por un momento en el mito de Awoinorü'pö, son los animales 
de la noche, que son predadores de los seres humanos. El atributo de la noche refiere a la oscuridad y a esta parte del esak de los animales. El kanaima es un servidor para estos entidades del estrato aiyan. Por ello es percibido como un enemigo por el mundo humano y se lo puede denominar como un anti-humano.

Por otro lado, los kanaimaton son necesarios para mantener el equilibrio en el multiverso Pemón. Escuché esta idea por primera vez durante mis entrevistas con diferentes indígenas Pemón. La lógica se encuentra en los trabajos del pia'san y del kanaima. Para especificar este asunto debe subrayarse que en la cosmología Pemón cualquier pia'san puede ser un kanaima también. Todos los pia'san conocen el mundo de los kanaimaton y pueden actuar como un kanaima. La última gran pia'san pachi (chamana) de la área de Kamarata, de nombre Usankoro, fue muerta por ser un kanaima según las explicaciones Pemón. Sin embrago, debe notarse que un kanaima no es automáticamente un pia'san. Como hemos visto en el ejemplo de la niña existen entidades (humanas) que tienen un conexión física con el estrato de aiyan. Entonces puede concluirse que por lo menos hay dos diferentes clases de kanaima. La primera clase contiene los que solamente saben y practican el estado de kanaima, y la segunda son los pia'san malos que conocen y aplican las prácticas de kanaima.

Para obtener estatus de pia'san es necesaria una larga formación educativa. Durante este proceso largo el chaman profesor realiza una prueba con sus novicios. La tarea, por ejemplo, es matar algunos pájaros en interacción con los espíritus u otras entidades no-humanas como las del estrato de aiyán. Los novicios que no ejecutan esta instrucción son categorizados como chamanes buenos, los otros son chamanes malos porque se conectaron con la parte de las energías negativas. Sin embargo estos pia'san también pueden curar, mientras un kanaima de la primera clase no puede actuar como un curandero. Además los pia'san buenos también pueden transformarse y actuar como un pia'san malo.

Finalmente puede constatarse que los kanaimaton y/o los pia'san malos sacrifican carne humana ${ }^{14}$ en el estrato aiyan. Este sacrificio garantiza un equilibrio en el colectivo de las entidades (humanas y no humanas) del multiverso Pemón. En otras palabras, el que mata 
contribuye para que un pia'san bueno pueda curar y negociar con las entidades del pia daktai y del estrato aiyan. Desde este punto de vista la perspectiva es otra. La reciprocidad exige que el pia'san 'bueno' nunca mate apenas, dejando el trabajo 'sucio' a los kanaimaton y/o pia'san 'malos'.

En este punto debemos recordar que el sacrificio existe en otros estratos también, como en la comida de los maikok o en la carne que dejan los cazadores para ayuk después de la cacería. Ayuk es el espíritu de una planta, que ayuda a los cazadores. Por esta razón debe dejársele la primera presa cazada en la selva. En caso que el cazador no cumpla esa exigencia de reciprocidad tendrá su cuerpo cubierto con zarpullido.

Los dos tipos (pia'san y kanaima) son temidos por los seres humanos. Este miedo produce una reacción entre algunos grupos de personas. Como vimos, existen grupos especiales que se denominan a si mismos como cazadores de kanaima. Mientras el pia'san siempre es temido y respetado por su gran conocimiento y poder, el kanaima tiene que generar prácticas para reducir las posibilidades de que lo maten. Una estrategia -la más pasiva- es que los kanaimaton que no son identificados no anuncian que sienten conexiones con el mundo del estrato de aiyan. Si se acumulan las acusaciones, ellos deciden vivir fuera de la comunidad en la sabana, solos o con su familia.

Existe otra estrategia más ofensiva, que es casi opuesta a la anterior. A través de amenazas, intimidaciones y humillaciones hacia las víctimas y sus familias el kanaima manifiesta sus intenciones y actos violentos.

De esta manera se debe leer la segunda categoría de amanawui que pueden describirse como cantos anti heroicos. La chamana Usankoro (Imagen 6) fue grabada en los años noventa del siglo XX. La canción registrada consiste en una pregunta para saber "si la mujer de la víctima esta llorando" mostrando que el kanaima confiesa el asesinato, sin sentir vergüenza, ofendiendo a la familia de la víctima. Nótese a su vez que el término yakon significa hermano. En este contexto es usado para referir a un Pemón conocido pero que no tiene necesariamente lazos de consanguinidad con el kanaima. 
1. Yakon pachi karawü nase? 1. ¿Esta llorando la mujer de mi hermano?

Imagen 6: Canto de amanawui, cantante Usankoro (región de Kamarata, 1992)

Fonte: Transcripción/Interpretación: Balbina Lambos

Otra intención del kanaima al entonar canciones como la de Usankoro (Imagen 6) es la confirmación del asesinato y/o sacrificio a las entidades del estrato aiyan.

\section{Conclusión}

Los cantos de los kanaimaton pueden clasificarse en dos categorías. La primera categoría contiene los cantos de los estilos de oarebá y/o warepán con la típica palabra aiyan. Este término sirve para construir una conexión con los mundos no humanos como el de los mawariton en las montañas y aguas y también para interactuar con las entidades del estrato de aiyan.

La información de Whitehead (2002) diciendo que los kanaimaton se conectan con un mundo de Makunaima no fue confirmada por los especialistas Pemón consultados. Para estos grupos el héroe cultural desapareció después de sus acciones en el tiempo mítico a la Guyana inglesa y nunca apareció otra vez.

En este contexto se nota la conexión de los kanaimaton con las entidades del estrato de aiyan, aunque no sean claramente nombradas. El único índice se encuentra en el mito de Awoinorü'pö en que aparecen los animales de la noche. Otra referencia es la conexión física entre todas las entidades kanaimaton.

Es importante mencionar que los especialistas explican la necesidad de la existencia kanaima para manejar el equilibro del multiverso Pemón. Aunque debemos recordar también que el grupo de los orekotón rechaza esa hipótesis diciendo que las prácticas de los rituales de areruya y cho'chiman son suficientes.

Los cantos descriptos se practican durante dos fases del estado kanaima. La primera fase se caracteriza por cantos para producir una conexión con el estrato de aiyan. En esta fase del ritual, marcado por la persecución y los ataques a la víctima, no se canta aunque sí se usa el 
tarén, ejecutado por el kanaima. La fase liminal del ritual se caracteriza por el suspenso generado cuando aún no se sabe si el kanaima caliente, loco, transformado en un monstruo y sin alma será capaz de recuperar su alma para re-transformarse. En esta fase se usa un canto del género amanawui. Otro posibilidad es el consumo de una parte del muslo de la carne de la víctima.

Estas características implican dos asuntos críticos en las discusiones de la etnología indígena actual.

El primer asunto se refiere a los conceptos de perspectivismo/ sonorismo (Lewy, 2017). La diferencia se encuentra en la parte de la función del canto en comparación con la formula mágica (tarén). La palabra cantada en su estructura antropomorfa transciende los estratos del multiverso. Por ejemplo, a través de los cantos del chamán, su alma puede conectarse con espíritus específicos. El mismo proceso se da cuando los cazadores cantan un parichara o los pescadores cantan un tukuik. En ambos casos los cantantes se conectan con el esak (dueño) de los animales. El kanaima canta para entrar e interactuar con las entidades del estrato de aiyan, específicamente para recuperar su alma, calmarse y re-transformarse en un ser humano. Debe constatarse que la percepción visual del otro no es importante, porque todas las entidades se comunican a través del canto (Lewy, 2012). La performance de las fórmulas mágicas, que son murmuradas y sopladas a la víctima, cambian la percepción visual de ésta sin transformar el cuerpo del asesino en la primera fase del ataque. Es decir, esa performance auditiva cambia la perspectiva de las víctimas, el kanaima no se transforma físicamente.

El segundo asunto trata del concepto de kanaima como un anticoncepto del animismo y chamanismo.

Mientras el chamán separa el alma de su cuerpo, para que su alma pueda andar por el mundo de los espíritus, el kanaima separa el cuerpo de su alma para matar en su físicalidad sin alma. En concreto significa que el kanaima necesita su cuerpo para matar y cumplir los órdenes de las entidades del estrato de aiyan.

En otras palabras, el kanaima puede viajar con su alma y cambiar la vista de los seres humanos a través de su tarén, pero en el momento de matanza el pierde el control de su alma que se fue. Así se puede 
resumir que el concepto de estado kanaima es un concepto de un "físicalismo" indígena que se opone al concepto de animismo en que todas las entidades están definidas por la cualidad de su interioridad (alma o almas). Al kanaima le falta justamente su alma cuando está en estado kanaima. Debe subrayarse otra vez, que la recuperación del alma se realiza a través del canto o por un acto antropófago.

Esta fue la explicación que me dio Florinda durante el diálogo con que abrí este texto sobre los cantos de kanaima. Vamos a cerrarlo ahora refiriendo al pensamiento de Theodor Koch-Grünberg (1923, p. 218) acerca del kanaima: "'Der Kanaime ist gar kein Mensch', so heißt es ${ }^{15 "}$.

\section{Notas}

1 Theodor Koch-Grünberg visitó a los Makuxí y Taurepán y también trabajó con un informante Arekuna. Los Taurepán y Arekuna se autodenominan como Pemón incluyendo el grupo de los Kamarakotos también. Los grupos viven en la Gran Sabana (Venezuela), en Guyana y en el norte de Brasil (Estado Roraima).

$2[\ldots]$ he is also believed to be able to detach his vital force, or spirit, from his body and to perform his killing whilst disembodied. Akawaio and Pemong say that he can thus be seen as an ordinary person in one place that his inherent evil, and 'bad mind' dictates. There are special plants substances (notable of the kumi species, which are reeds and coarse grasses) which enable Itoto (Kanaima) to travel many miles within a few minutes. This is consonant with a general belief that all material forms of life consist of a body and an indwelling vital force or spirit (Akawaio, akwalu, Pemong, ekatong). The vital force leaves its body temporarily during states of unconsciousness: in dreams, when fainting, or in coma. A shaman deliberately detaches his spirit when hallucinated by tobacco. Itoto (Kanaima) can voluntarily detach his vital force and send it to kill, but he may also send it into another creature, a dog, bird, jaguar, deer, and so forth, using its body as a disguise. (Butt Colson, 2001, p. 223)

3 David Thomas (1982, p. 235; Halbmayer, 2010, p.161) presenta el mismo argumento de que los kanaimaton no atacan a miembros de su propia familia.

4 Kanaima is more ancient than piya, more ancient than warfare. We searching for our food that is all. That is all it is. We look at someone and see something sweet to suck and all that [ritual procedures], is for food. It boost your spirit up, up to go far in kalawali. (Whitehead, 2002, p. 109)

5 Existe otra versión del mito publicada con el título: "Costumbres de Awoinorü'pö" (Armellada, 2013, p. 34, II). La versión que transcribimos aquí -que es una traducción del pemón- fue retirada del libro de Cesáreo de Armellada, (2013, I: p. 229.). Mantuvimos el texto como fue publicado por Armellada.

6 If the grave site is discovered, a stick is inserted through the ground directly into the cadaver, then the stick is retracted and the maba (honey $\square$ like) juices sucked off. The juices of putrefaction are said to taste like honey because the grave is itasted ‘ with the help of a stick, used in the same way as when eating honey from a 
hive. The effect of maba is both psychotropic and morphic: the satisfied Kanaima can, like Jaguar, now sleep sated and dream of being Man, but the dream is also a reality since tasting the maba thereby transforms Jaguar into Man again. So a Kanaima will not eat human food after a kill until he has tasted the divine food of the grave, and indeed he must taste the maba if the dangerous divine force of Lord Jaguar is to be purged and this return to the domain of the human achieved.

7 El término orekotón refiere a todas las entidades que son parte de los rituales de areruya/cho'chiman. Se incluyen las almas de fallecidos de participantes de los rituales y los miembros vivos que practican areruya/cho'chiman y los mensajeros que son por partes santos de la iglesia católica (San Miguel, San Francisco, Jesús) o las almas de líderes del ritual fallecidos (Auka, Püreri pachi). Finalmente Dios es parte de todo el colectivo también [...].

8 Ernst Halbmayer (2010) presenta todas las informaciones accesibles y muestra un imagen bien detallado sobre el multiverso Pemón.

9 Auka tiene muchas significados, entre otros era una persona que fundó el areruya en la área de los Arekuna. También es la luz que vean los participantes de los rituales de orekotón cuando entran en trance, es decir en el momento en que se separa el alma al cuerpo (Lewy, 2011)

${ }^{10}$ It seems therefore, that this particular Kapon and Pemon activity related especially to the basis of the food chain, to the vegetable growth and cycle of flowering, fruiting and seeding which supports the animal kingdom at large - upon which all depend, whether directly (by eating fruits) or indirectly (by eating those who eat the fruits).

11 Otra forma de baile en el contexto orektón parecido al cho'chiman.

12 El texto esta transcripto pero no publicado por el momento por falta de más aclaraciones por parte de los especialistas Pemón. Quiero agradecer a Jesús García por el acceso al material.

13 No publico metadatos por motivos éticos.

14 Según mis informantes la carne humana tiene que ser de un/a indígena. Criollos y blancos son detestado por los kanaimaton por su hedor. Tampoco sirve matar a cualquier humano no indígena, ya que no son parte del colectivo humano y no humano del multiverso Pemón.

15 “"El Kanaime no es un ser humano', así se dice." (Traducción del autor)

\section{Referências}

ARMELLADA, Cesareo de. Tauron Panton: Cuentos y Leyendas de la Etnia Pemón. Caracas: UCAB, 2013. II Tomos.

ARMELLADA, Cesáreo de. Diccionario Pemón: Pemón-Castellano, Castellano-Pemón. Caracas: UCAB, 2007.

BUTT COLSON, Audrey. Itoto (Kanaima) as death and Anti-Structure. In: RIVAL, Laura M.; WHITEHEAD, Neil L. (Org.). Beyond the Visible and the Material. Oxford: Oxford University Press, 2001. p. 221-235. 
BUTT COLSON, Audrey; ARMELLADA, Fray Cesáreo de. The Pleiades, Hyades and Orion (Tamökan) in the conceptual and ritual system of Kapon and Pemon groups in the Guiana Highlands. Scripta ethnologica, [S.l.], (suppl. 9), p. 153-200, 1989.

CIVRIEUX, Marc de. Watunna: An Orinoco Creation Cycle. San Francisco: North Point Press, 1980.

HALBMAYER, Ernst. Kosmos und Kommunikation: Weltkonzeptionen in der südamerikanischen Sprachfamilie der Cariben. Facultas wuv: Wien. 2010. 2 V.

KOCH-GRÜNBERG, Theodor. Vom Roroima zum Orinoco: Ergebnisse einer Reise in Nordbrasilien und Venezuela in den Jahren 1911-1913. Stuttgart: Strecker und Schröder, 1923. 3 v.

LEWY, Matthias. Die Rituale areruya und cho'chiman bei den Pemón (Gran Sabana/ Venezuela). Tese (Doutorado) - Berlin: Freie Universität Berlin, 2011.

LEWY, Matthias. Different "Seeing" - Similar "Hearing". Ritual and Sound among the Pemón (Gran Sabana/Venezuela). Indiana, [S.l.], v. 29, p. 53$71,2012$.

LEWY, Matthias. About Indigenous Perspectivism, Indigenous Sonorism and the Audible Stance. Approach to a Symmetrical Auditory Anthropology. El Oído Pensante, [S.l.], v. 5, n. 2, p. 1-22, 2017.

SCHOMBURGK, Robert Hermann. Reisen in Guiana und am Orinoko während der Jahre 1835-1839. Leipzig: [s.n.], 1841.

SCHOMBURGK, Richard. Reisen in bis 1844: Zweiter Theil. BritischGuiana in den Jahren 1840. Leipzig: [s.n.], 1848.

THOMAS, David J. Order Without Government: The Society of the Pemon Indians of Venezuela. Urbana: University of Illinois Press, 1982.

WHITEHEAD, Neil L. Dark Shamans. Kanaima and the poetics of violent death. Durham: Duke University Press, 2002.

Recebido em 23/12/2017

Aceito em 30/04/2018 